\title{
Enhancer-driven chromatin interactions during development promote escape from silencing by a long non-coding RNA
}

\author{
Lisa Korostowski, Anjali Raval, Gillian Breuer and Nora Engel*
}

\begin{abstract}
Background: Gene regulation in eukaryotes is a complex process entailing the establishment of transcriptionally silent chromatin domains interspersed with regions of active transcription. Imprinted domains consist of clusters of genes, some of which exhibit parent-of-origin dependent monoallelic expression, while others are biallelic. The Kcna1 imprinted domain illustrates the complexities of long-range regulation that coexists with local exceptions. A paternally expressed repressive non-coding RNA, Kcnq1ot1, regulates a domain of up to $750 \mathrm{~kb}$, encompassing 14 genes. We study how the Kcnq1 gene, initially silenced by Kcnq10t1, undergoes tissue-specific escape from imprinting during development. Specifically, we uncover the role of chromosome conformation during these events.

Results: We show that Kcnq1 transitions from monoallelic to biallelic expression during mid gestation in the developing heart. This transition is not associated with the loss of methylation on the Kcnal promoter. However, by exploiting chromosome conformation capture (3C) technology, we find tissue-specific and stage-specific chromatin loops between the Kcnq1 promoter and newly identified DNA regulatory elements. These regulatory elements showed in vitro activity in a luciferase assay and in vivo activity in transgenic embryos.

Conclusions: By exploring the spatial organization of the Kcnq1 locus, our results reveal a novel mechanism by which local activation of genes can override the regional silencing effects of non-coding RNAs.
\end{abstract}

Keywords: Imprinting, non-coding RNAs, Kcnq1ot1, Kcnq1, chromosome conformation capture (3C)

\section{Background}

Genomic imprinting is a transcriptional regulatory mechanism that results in parental-specific gene expression. Over the past two decades, many mechanistic insights have emerged from the study of such loci as the H19/Igf2 domain [1]. However, most imprinted loci are much more complex and exhibit tissue-specific as well as stage-specific imprinting. Many significant questions remain concerning the regulatory mechanisms governing such extended domains. For example, how genes that are monoallelic can coexist interspersed with others that exhibit partial or full biallelic expression is still not understood. The prevalent hypotheses for the

\footnotetext{
* Correspondence: noraengel@temple.edu
Fels Institute for Cancer Research \& Molecular Biology \& Department of

* Correspondence: noraengel@temple.edu Biochemistry, Pharmacy Building, Room 201, Temple University School of Medicine, Philadelphia, PA 19104, USA
}

(c) 2011 Korostowski et al; licensee BioMed Central Ltd. This is an Open Access article distributed under the terms of the Creative Commons Attribution License (http://creativecommons.org/licenses/by/2.0), which permits unrestricted use, distribution, and reproduction in any medium, provided the original work is properly cited. appearance of imprinting imply that, although advantader genes that came under its influence. Thus, it is likely that mechanisms emerged to bypass the effects of allelic silencing.

The Kcnq1 domain consists of at least ten genes exhibiting parental allele-specific expression [2], interspersed with five genes that are biallelically expressed. The key regulatory element, KvDMR, is a CG-rich promoter for a long, non-coding RNA [3]. The paternal copy of the KvDMR is hypomethylated and active, resulting in production of a 90-kb non-coding RNA (ncRNA), Kcnq1ot1. Transcription of the Kcnq1ot1 RNA has cissilencing effects on the neighboring genes, spanning a region of $750 \mathrm{~kb}$ in the placenta and $400 \mathrm{~kb}$ in the embryo. The KvDMR is methylated on the maternal allele and as a consequence, maternal promoter activity 
is inhibited. As the ncRNA is not produced maternally, most genes within the domain are free to be expressed from that chromosome (Figure 1).

Recent experiments have strongly suggested that the silencing mechanism of Kcnq1ot1 RNA involves a spreading activity in cis, recruitment of Polycomb group proteins and physical compaction of the whole domain [4-6]. However, the presence of the ncRNA is not uniform throughout the region [4], leaving open the question of how this relates to biallelic expression of some genes.

Genes such as Trpm5 are constitutively biallelic both in the embryo and placenta. The Kcnq1 gene has a more complex pattern: it is monoallelic and ubiquitously expressed in early embryos, but the paternal copy is activated in conjunction with the acquisition of a tissuerestricted expression pattern established by mid gestation, that is, in the heart, kidney and brain [7]. Thus, the Kcnq1 domain illustrates the regulatory challenges that must be met in a complex imprinted domain.

How do genes such as Kcnq1 achieve tissue-specific escape from imprinting? One possible explanation is that the Kcnq1 promoter is exceptionally strong and that as tissue-specific factors become expressed and bind to it, the silencing effect of Kcnq1ot1 is overcome. Alternatively, tissue-specific enhancers that become active may override the effects of ncRNAs. An additional possibility is that boundary elements serving as barriers to the spread of the ncRNA may exist within the Kcnq1 gene. These proposed mechanisms are not mutually exclusive.

None of the regulatory elements that account for the complex patterns of gene expression in this region have been identified. We used an optimized approach for identifying novel regulatory DNA elements and to determine their role in promoting escape from silencing. We investigated the mechanism of Kcnq1 reactivation by determining the in vivo spatial organization of the Kcnq1 domain with chromosome conformation capture (3C) assays [8,9]. We present evidence for the role of specific DNA interactions by chromatin looping as a mechanism for acquisition of tissue-specific expression. These contacts occur during the developmental window in which the paternal allele of Kcnq1 escapes silencing by Kcnq1ot1. To determine if the regions contacted predominantly by the $K c n q 1$ promoter are regulatory elements, we integrated comparative genomics data and publicly available genome-wide chromatin immunoprecipitation (ChIP) profiles and identified DNA sequences that are candidates for modulating gene activity. Candidates were tested in vitro and in vivo and several elements were identified as active transcriptional enhancers. Their role in overriding RNA-mediated repression is discussed.

\section{Results}

Kcnq1 RNA transitions from monoallelic to biallelic expression during development

Congenital long QT syndrome type I is a cardiac disorder in which defects in KCNQ1, a voltage-gated potassium channel, result in serious cardiac arrhythmias [10]. There is a wide range of phenotypes, with some individuals remaining mostly asymptomatic and others presenting severe symptoms. Understanding the epigenetic profile of Kcnq1 expression during cardiac development will aid in understanding the molecular mechanisms underlying the phenotypic variability. We determined Kcnq1 expression levels during development to pinpoint the exact timing of the switch from monoallelic to biallelic expression (Figure 2A). Allele-specific reverse transcription (RT)-PCR showed that Kcnq1 switched from a monoallelic to biallelic pattern between E13.5 and E14.5.

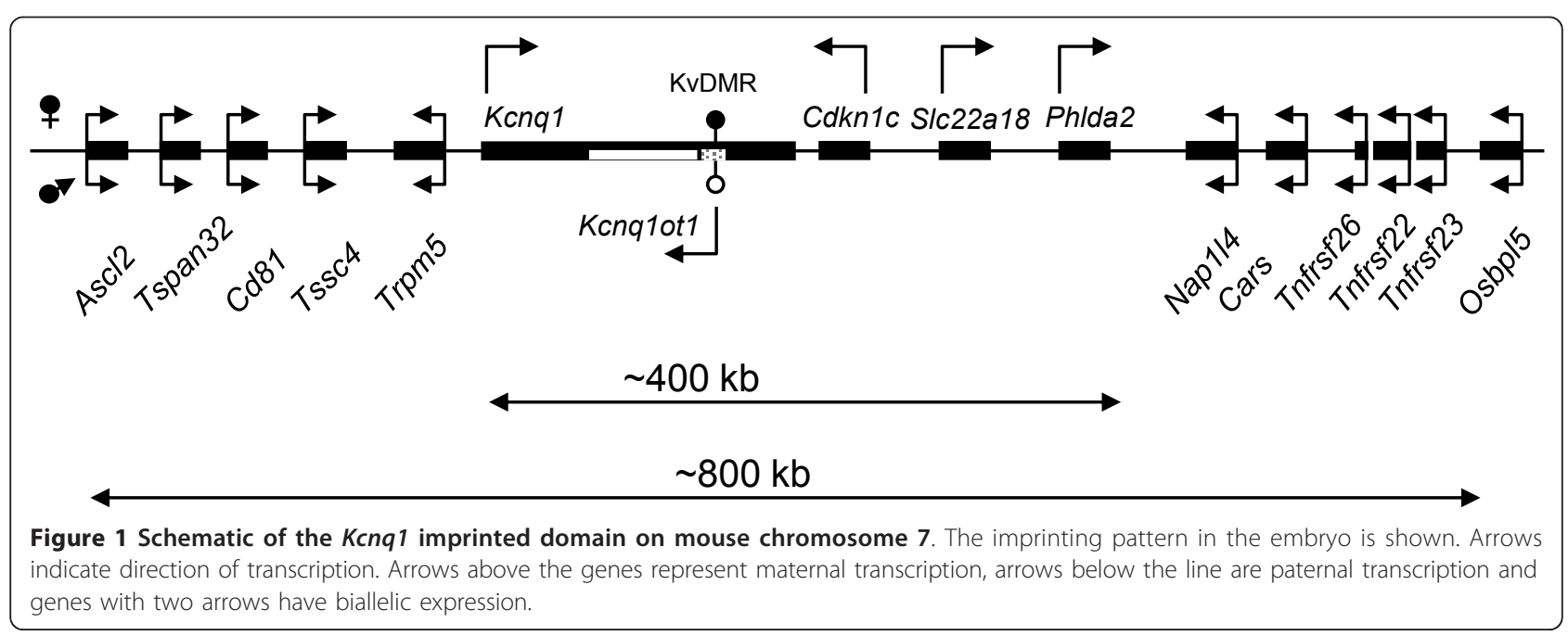




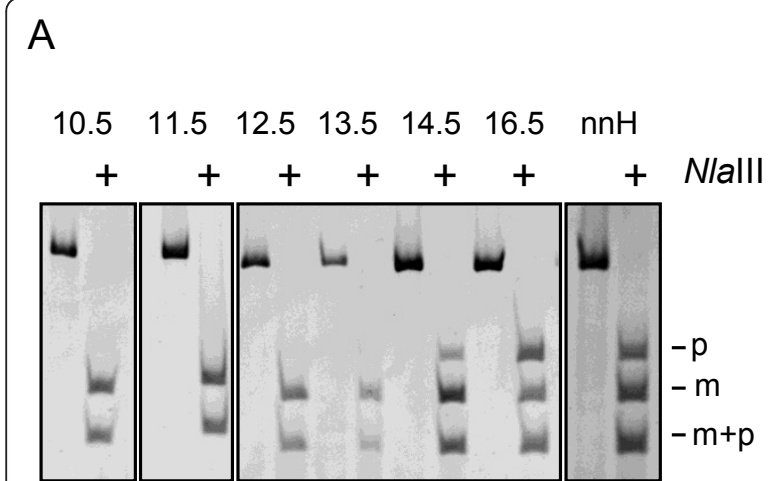

B

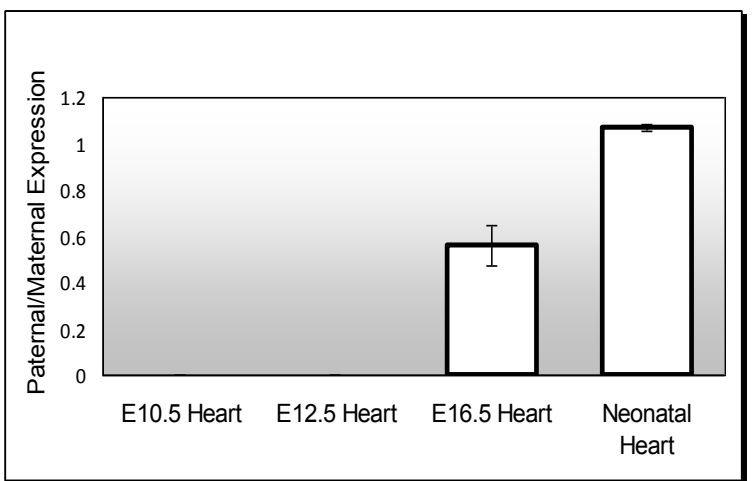

C

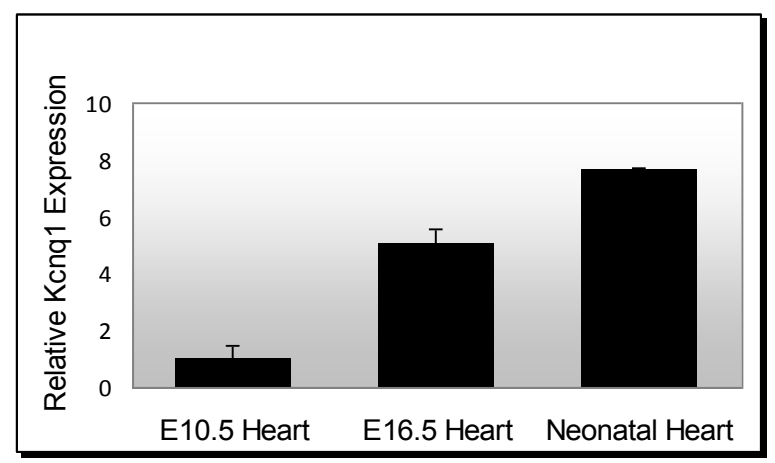

$\mathrm{D}$

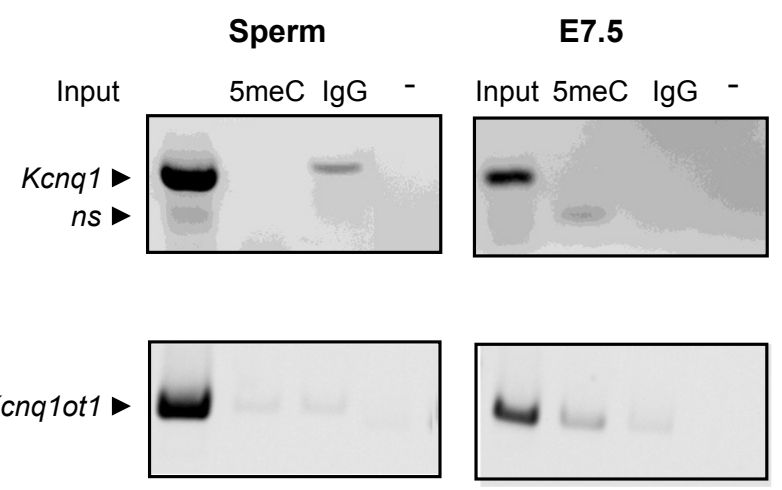

Figure 2 (A) Developmental imprinting pattern of Kcnq1. Allele-specific expression of Kcnq1 as assayed by reverse transcription (RT)-PCR and restriction digest with Nlalll on E10.5, 11.5, 12.5, 13.5, 14.5, 16.5 and neonatal heart $(\mathrm{nnH})$ from F1 hybrid B6(CAST7) $\times$ C57BL/6J crosses. Digestion products specific for B6(CAST7) (maternal) and C57BL/6J (paternal) alleles are indicated. Positive signs (+) denote addition of Nlalll to the RT-PCR product. (B) Quantification of relative paternal-specific and maternal-specific expression during development. (C) Kcna1 RNA abundance during stages of development in which the imprinting pattern switches from monoallelic to biallelic, as assayed by real-time PCR. (D) Methylated DNA immunoprecipitation (MeDIP) analysis of the Kcnq1 and Kcnq1ot1 promoter regions in sperm and 7.5 days post coitum (dpc) embryos. $5 \mathrm{meC}$ lane = DNA precipitated by antibody against methylated cytosine; lgG = non-specific immunoprecipitation; Input = DNA before immunoprecipitation; - = no antibody control. Specific bands for Kcnq1 and Kcnq1ot1 are indicated; NS = non-specific amplification product. The Kcnq1ot1 promoter is methylated maternally in $7.5 \mathrm{dpc}$ embryos and unmethylated in sperm, thus serving as a positive control for immunoprecipitation of methylated DNA in E7.5 DNA and a negative control in sperm DNA.

Expression emanating from the paternal allele became progressively stronger, reaching levels equal to the maternal allele (Figure 2B). To determine whether the activation of the paternal Kcnq1 allele was accompanied by an increase in total Kcnq1 RNA abundance, quantitative (q)PCR on total RNAs from these stages was performed. Whereas the transition from monoallelic to biallelic expression is expected to double RNA abundance, the total cellular RNA levels increased eightfold (Figure 2C), suggesting that other enhancing mechanisms are involved in achieving the final RNA levels.

One possible explanation for escape of silencing by the paternal Kcnq1 allele is that expression is initiated from an alternative start site. However, we did not observe any alternative transcripts after the transition from monoallelic to biallelic expression, thus excluding this hypothesis (data not shown). We then speculated that, because the transcriptional start site (TSS) at exon 1 lies within a strong CpG island, it could be paternally methylated in the early embryo and become demethylated upon activation. To determine if the $\mathrm{CpG}$ island was methylated during spermatogenesis or early embryogenesis, we performed methylated DNA immunoprecipitation (MeDIP) on sperm and embryos at 7.5 days post coitum (dpc) (Figure 2D). No DNA methylation was detected on the paternal allele, suggesting that the promoter is protected from primary (gametic) and secondary (post fertilization) methylation, even though it is silent at these stages. Furthermore, as opposed to the secondary methylation of the upstream Cdkn1c gene orchestrated by Kcnq1ot1, the silencing effect of the ncRNA on Kcnq1 is not mediated by DNA methylation. 


\section{Kcnq1 exhibits tissue-specific changes in chromatin conformation}

Three-dimensional conformation of chromatin plays a crucial role in bringing widespread regulatory elements into close proximity [11-13]. 3C technology detects interactions between DNA regions by in vivo crosslinking of chromatin and PCR with primers specific for novel junctions created by ligation of sequences that were in close proximity through specific interactions. To determine if the Kcnq1 promoter associated with specific DNA sequences in the neonatal heart, we performed an unbiased, systematic $3 \mathrm{C}$ scan extending $12.6 \mathrm{~kb}$ upstream of the promoter, spanning the $320 \mathrm{~kb} \mathrm{Kcnq1}$ gene and $31.3 \mathrm{~kb}$ downstream of the last exon. Our strategy was to anchor the $3 \mathrm{C}$ assays with an invariant primer at the Kcnq1 promoter (Figure 3A, white arrowhead) and variable primers located in restriction fragments across the domain (Figure 3A, fragments tested are numbered from 1 to 26). Strong and highly specific interactions were detected between the Kcnq1 promoter and fragment 4 (Figure $3 \mathrm{~B}$, orange line, peak at $-8 \mathrm{~kb}$ upstream of Kcnq1). Additionally, there were weaker but specific contact with fragments 11 and 12 (Figure 3B, orange line, peak in intron 1).

To see if this profile was tissue specific, we performed $3 \mathrm{C}$ in neonatal brain, a tissue in which Kcnq1 is highly expressed and also biallelic. The interaction profile observed in the brain was distinct from that of the heart. The Kcnq1 promoter strongly associated instead with fragment 2 (Figure 3B, blue line, peak at $-12 \mathrm{~kb}$ ) and showed no contact with fragment 12 in the intron. These data show that there are distinct three-dimensional conformations that regulate expression in each tissue and identify the contact regions as putative tissuespecific enhancers.

These results were verified by carrying out the reciprocal analysis, that is, $3 \mathrm{C}$ assays were anchored with a primer in fragment 12 of intron 1 (Figure 3C, white arrowhead at $+24 \mathrm{~kb}$ ). In the neonatal heart, we found increased association between this region and the Kcnq1 promoter, but also with fragment 4 , at $-8 \mathrm{~kb}$ upstream. We did not observe these contact points in neonatal brain (data not shown). In addition, reciprocal analyses with anchors at fragment 4 in neonatal heart (Figure $4 \mathrm{~B}$ ) and at fragment 2 in neonatal brain (Figure 4C) confirmed the interactions between these sequences with the Kcnq1 promoter region, underscoring the presence of tissue-specific conformations.

\section{Onset of loop formation between the Kcnq1 promoter} and putative enhancers correlates with the monoallelic to biallelic switch

We next looked for dynamic changes in the status of the chromatin fiber related to the escape from silencing of
Kcnq1 by testing the conformation in E11.5 hearts. At this stage, the paternal Kcnq1 allele is still silent (Figure $5)$. None of the interactions evident in the neonatal heart and brain between the Kcnq1 promoter and upstream regions was apparent in the embryos. However, there was a significant physical association of the promoter with a region in intron 1 , though restricted to fragment 11 (Figure 5B, yellow line). Thus, monoallelic expression is not dependent on contacts between the promoter and the putative upstream enhancers. However, onset of loop formation with those regions does correlate with the monoallelic to biallelic switch.

\section{Distinct developmental history of potential regulatory elements at the Kcnq1 locus}

To expand the epigenetic profile of Kcnq1 to earlier developmental stages, we performed locus-wide $3 \mathrm{C}$ assays on mouse ES cells (Figure 5B, blue line). None of the heart-specific contacts between the Kcnq1 promoter and regions upstream were detected, but the interaction with fragment 11 in intron 1 was already present. Thus, $3 \mathrm{C}$ assays show an interaction between the Kcnq1 promoter and a region in intron 1 that is already apparent in early stages of embryogenesis and becomes progressively stronger as development ensues. Additionally, the Kcnq1 promoter associates with upstream elements concomitantly with the transition from monoallelic to biallelic expression. These upstream elements partner with the Kcnq1 promoter in a tissue-specific fashion.

\section{Sequences identified by contact analysis have enhancer activity in vitro and in vivo}

A search of publicly available databases including the VISTA enhancer browser [14] revealed an intragenic element (mm183) with a heart-specific expression pattern in $11.5 \mathrm{dpc}$ transgenic mouse embryos. This element lies in fragment 10 , immediately upstream of the sequence contacted by the promoter in intron 1 . This strongly supports its role in the development of heartspecific transcription of Kcnq1. Examination of conservation profiles for the Kcnq1 region from the dCODE website (http://www.dcode.org) [15] revealed discrete evolutionarily conserved regions (ECRs) (parameters set to $70 \%$ identity with a minimum length of $100 \mathrm{bp}$ ) that were contained on restriction fragments that had been tested in the $3 \mathrm{C}$ substrates (Figure 3). By combining in silico analysis and published epigenomic profiles, including chromatin status [16] and occupancy of p300 [17], we selected ECRs that were good candidates for enhancers, focusing on the intergenic region upstream of the Kcnq1 gene, numbering them according to the $3 \mathrm{C}$ fragments they overlapped (Figure 6A,B). None of these ECRs were conserved in opossum, chicken, frog or fish. 


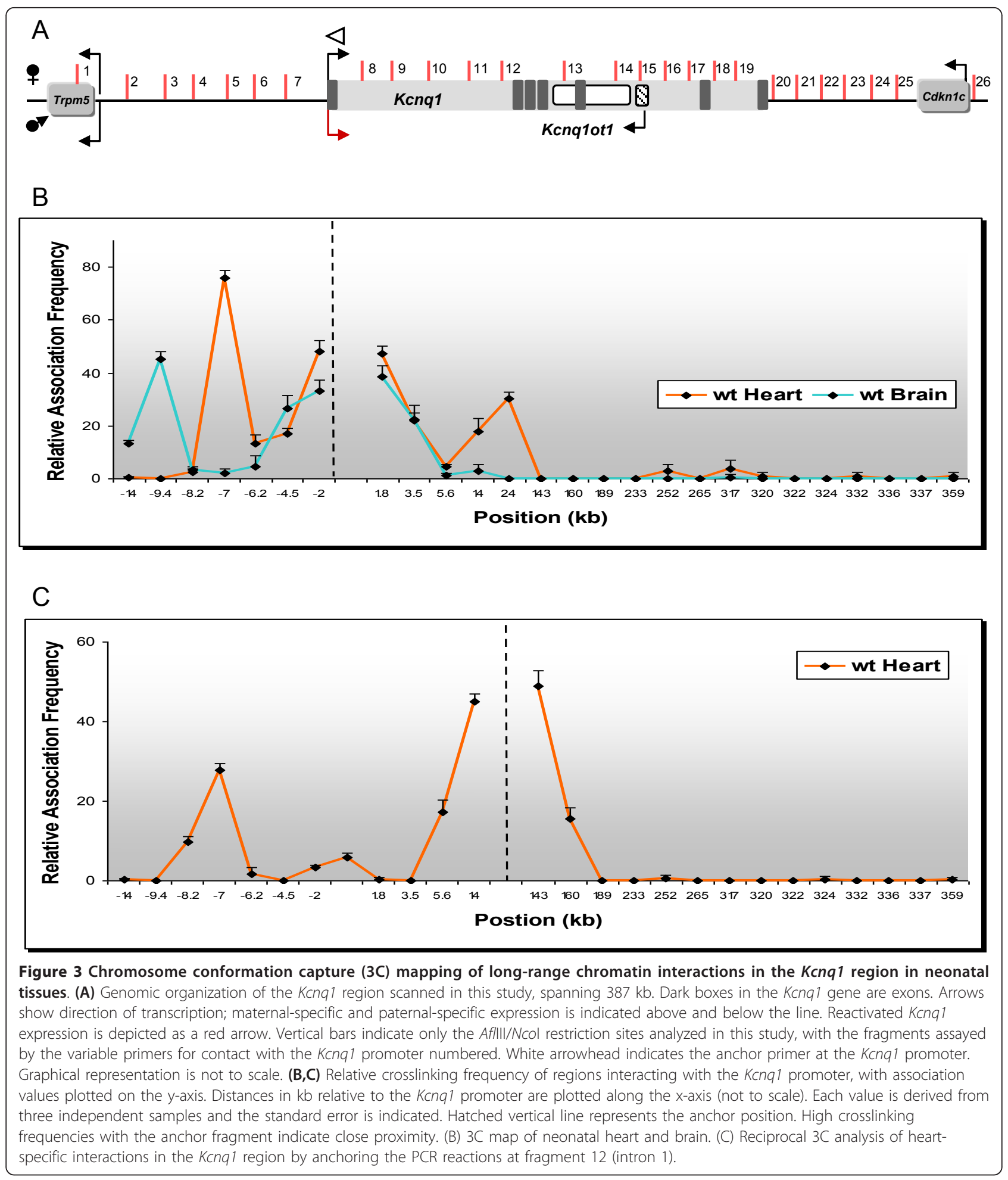

To determine whether these sequences had enhancer activity in vitro, we cloned the DNA fragments upstream of a luciferase reporter gene and tested enhancer activity by transient transfection into NIH-3T3 cells (Figure 6C). ECR 4, contained in fragment 4 that showed frequent colocalization with the Kcnq1 promoter in neonatal heart, had the most pronounced enhancer effect in vitro. ECR 2, which associated with the Kcnq1 promoter in the neonatal brain, did not show significant transcriptional activity in this assay, suggesting that fibroblasts 


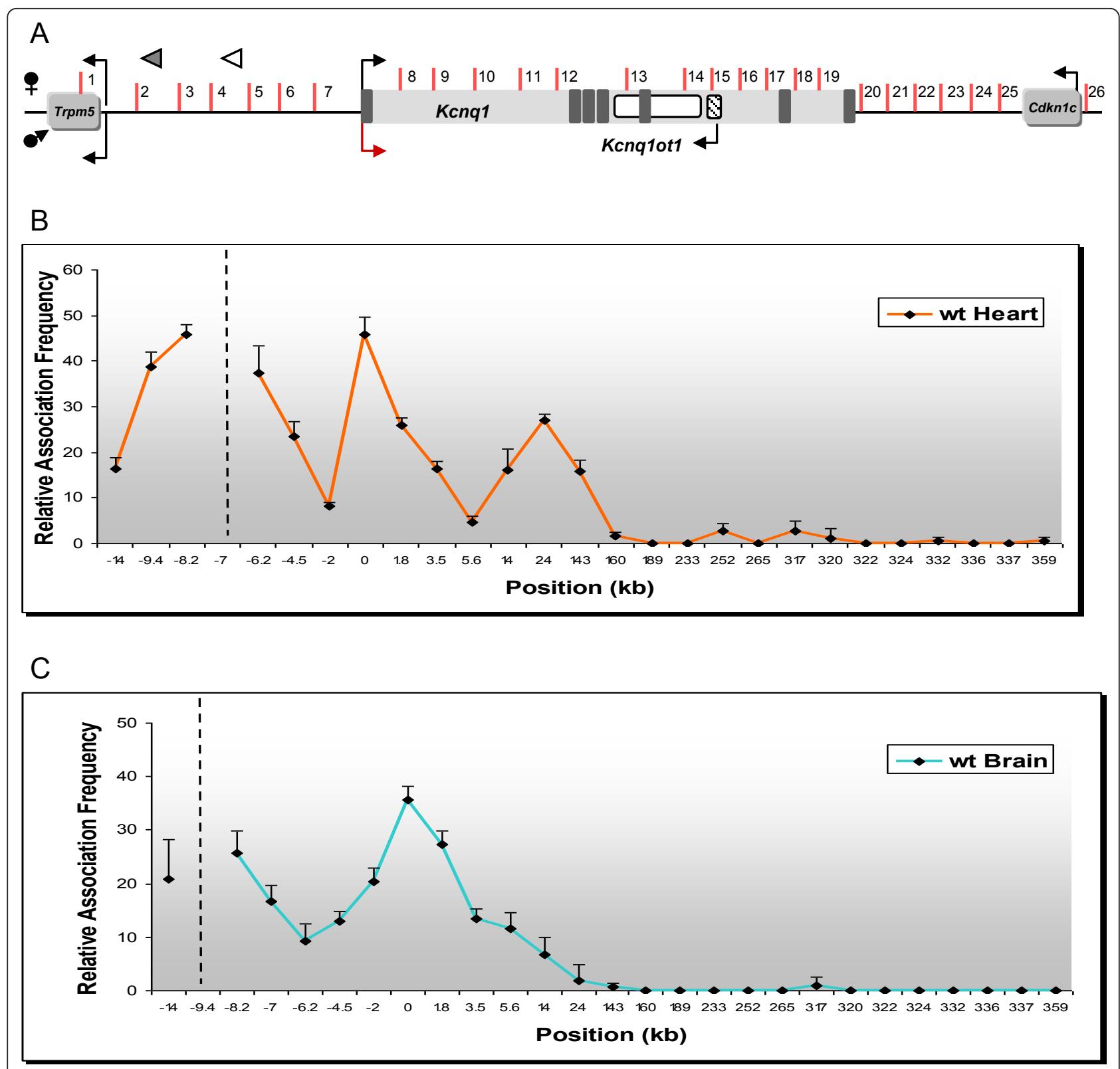

Figure 4 Reciprocal chromosome conformation capture (3C) scans anchoring at evolutionarily conserved regions. (A) Genomic organization of the Kcnal region scanned in this study, spanning $387 \mathrm{~kb}$. Dark boxes in the Kcnal gene are exons. Arrows show direction of transcription; maternal-specific and paternal-specific expression is indicated above and below the line. Reactivated Kcna 1 expression is depicted as a red arrow. Vertical bars indicate the Aflll/Ncol restriction sites analyzed in this study, with the fragments assayed with the variable primers for contact with the tissue-specific evolutionarily conserved regions (ECRs) numbered. White arrowhead, anchor primer for neonatal heart; gray arrowhead, anchor primer for neonatal brain. Graphical representation is not to scale. (B) Relative crosslinking frequency of regions interacting with ECR 4. The peaks correspond to the Kcnal promoter and fragment 12 within intron 1. (C) Relative crosslinking frequency of regions interacting with ECR 2. The peaks correspond to the Kcnq1 promoter and fragment 12 within intron 1. Hatched vertical lines represent the anchor position.

may not contain the factors required for brain-specific activity.

We next cloned several candidate sequences into an enhancer reporter vector and transgenic mouse embryos were generated. Several independent embryos were assessed for LacZ activity at 9.5 and $13.5 \mathrm{dpc}$.
Reproducible staining in hindbrain, forebrain and thoracic region was observed with ECR4 at 13.5 dpc (Figure $6 \mathrm{D})$, but none at $9.5 \mathrm{dpc}$ (data not shown), recapitulating the developmental expression pattern of Kcnq1. ECR2 directed expression to hindbrain and forebrain in the $13.5 \mathrm{dpc}$ embryos. 


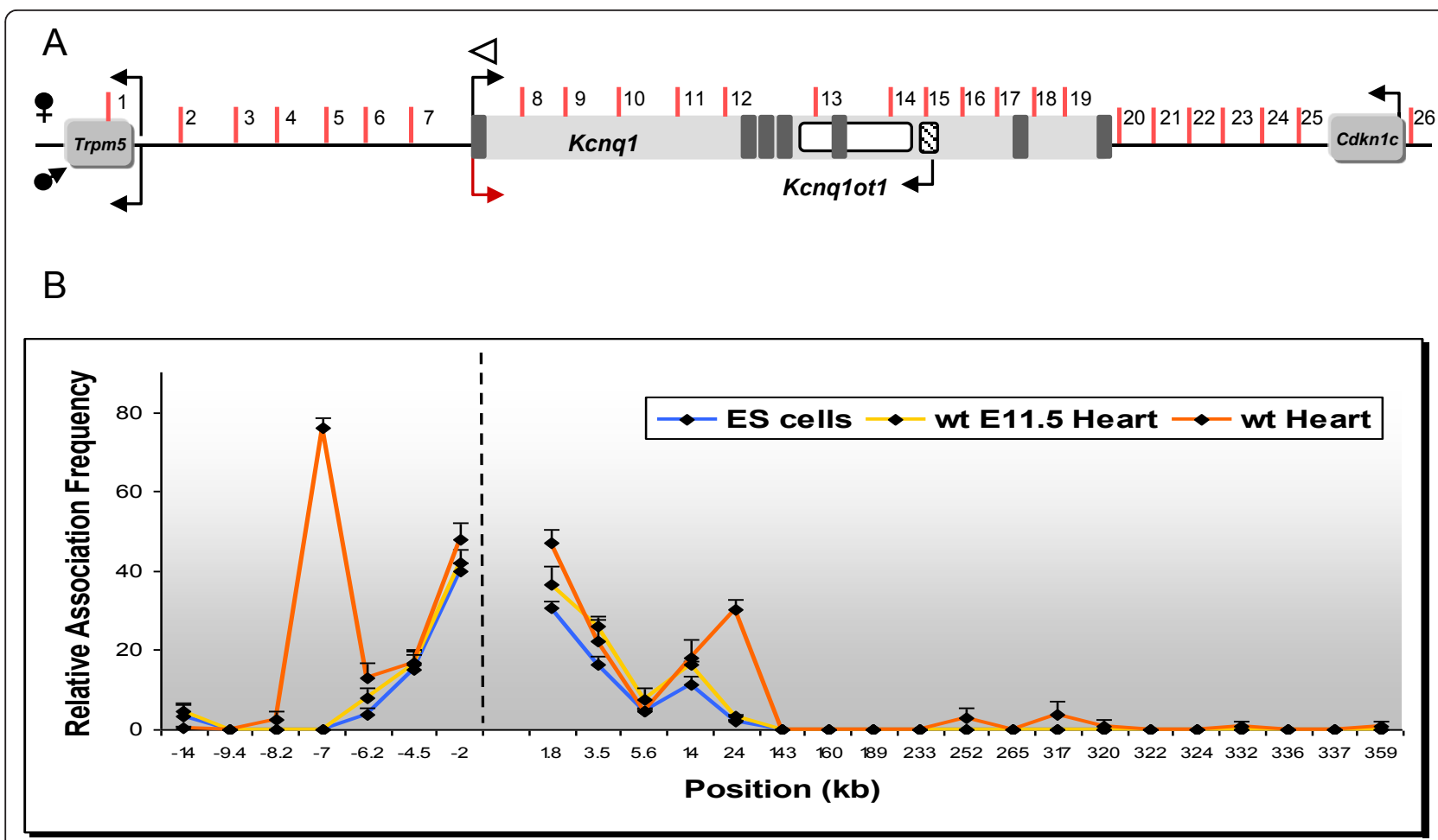

Figure 5 Developmental profile of chromatin interactions in the Kcnq1 region. (A) Genomic organization of the Kcnal region scanned in this study, spanning $387 \mathrm{~kb}$. Dark boxes in the Kcnal gene are exons. Arrows show direction of transcription; maternal-specific and paternalspecific expression is indicated above and below the line. Reactivated Kcnq1 expression is depicted as a red arrow. Vertical bars indicate only the Aflll/Ncol restriction sites analyzed in this study, with the fragments assayed with the variable primers for contact with the Kcna1 promoter numbered. White arrowhead indicates the anchor primer at the Kcnal promoter. Graphical representation is not to scale. (B) Developmental profile of interactions of the Kcnq1 promoter in ES cells, 11.5 days post coitum (dpc) heart, and neonatal heart. Relative crosslinking frequency of regions interacting with the Kcnal promoter, with association values plotted on the $y$-axis. Distances in $\mathrm{kb}$ relative to the Kcnal promoter are plotted along the x-axis (not to scale). Each value is derived from three independent samples and the standard error is indicated. Hatched vertical line represents the anchor position. High crosslinking frequencies with the anchor fragment indicate close proximity.

\section{Discussion}

Our data reveal a novel role for transcriptional enhancers, namely overcoming regional imprinting effects to permit escape from silencing and leading to biallelic expression. Thus, the evolution of enhancers in the mammalian genome to enable biallelic expression of some genes located near imprinted genes appears to be an essential adaptation to accommodate imprinting as a regulatory mechanism. By mapping the architecture adopted by a specific genomic region during development, we have found dynamic changes that counter the regional repressive effect of a long non-coding RNA, Kcnq1ot1. Specifically, escape from silencing of the Kcnq1 gene is accomplished by the activation of tissuespecific enhancers and involves local reorganization of higher order structure.

The biallelic expression of genes within the Kcnq1 domain is reminiscent of certain genes on the $\times$ chromosome that escape inactivation $[18,19]$, but differs in significant ways. Kcnq1 is initially silenced paternally but transitions to biallelic expression at a specific time point during development. In theory, tissue-specific enhancer activity could override silencing by Kcnq1ot1 by physically contacting the promoter and sequestering it from condensing factors recruited by the ncRNA. In fact, the data from the $3 \mathrm{C}$ assays that we present are highly supportive of such a model by showing that stage and tissue-specific conformations of chromatin bring the Kcnq1 promoter into physical contact with DNA sequences that are highly conserved and exhibit enhancer activity in vivo.

In fact, we could distinguish two types of contacts. Promoter interaction with an element in intron 1 ( $\mathrm{mm} 183)$ is already apparent in early stages of embryogenesis and becomes progressively stronger and broader as development ensues. This association foreshadows a later event in which the Kcnq1 promoter contacts tissue-specific upstream elements concomitantly with the reactivation of the paternal allele and the restriction of expression to specific organs. Indeed, previous studies on the chromatin marks in the region showed that, in contrast to other neighboring genes, the Kcnq1 promoter does not exhibit 

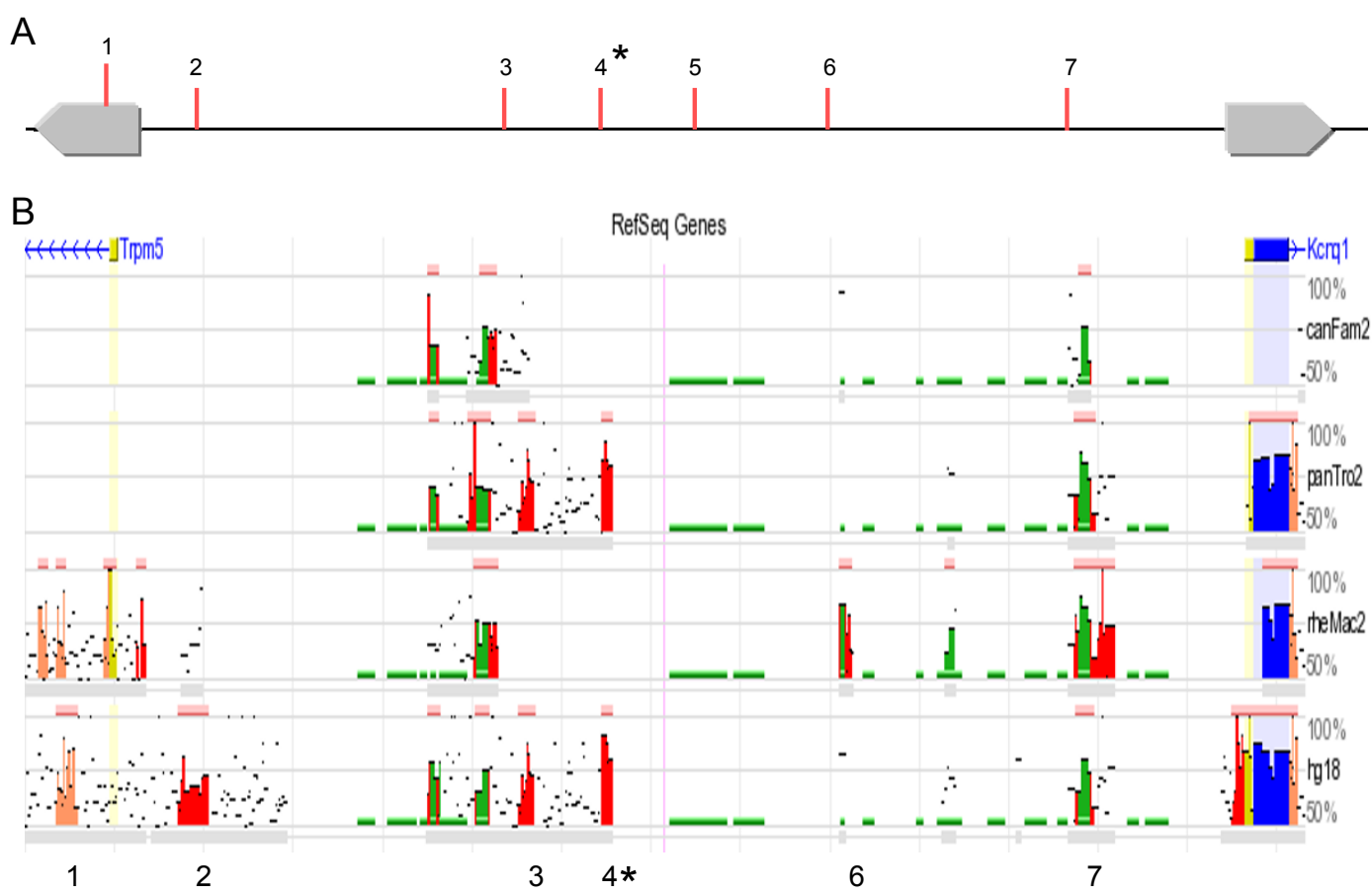

C

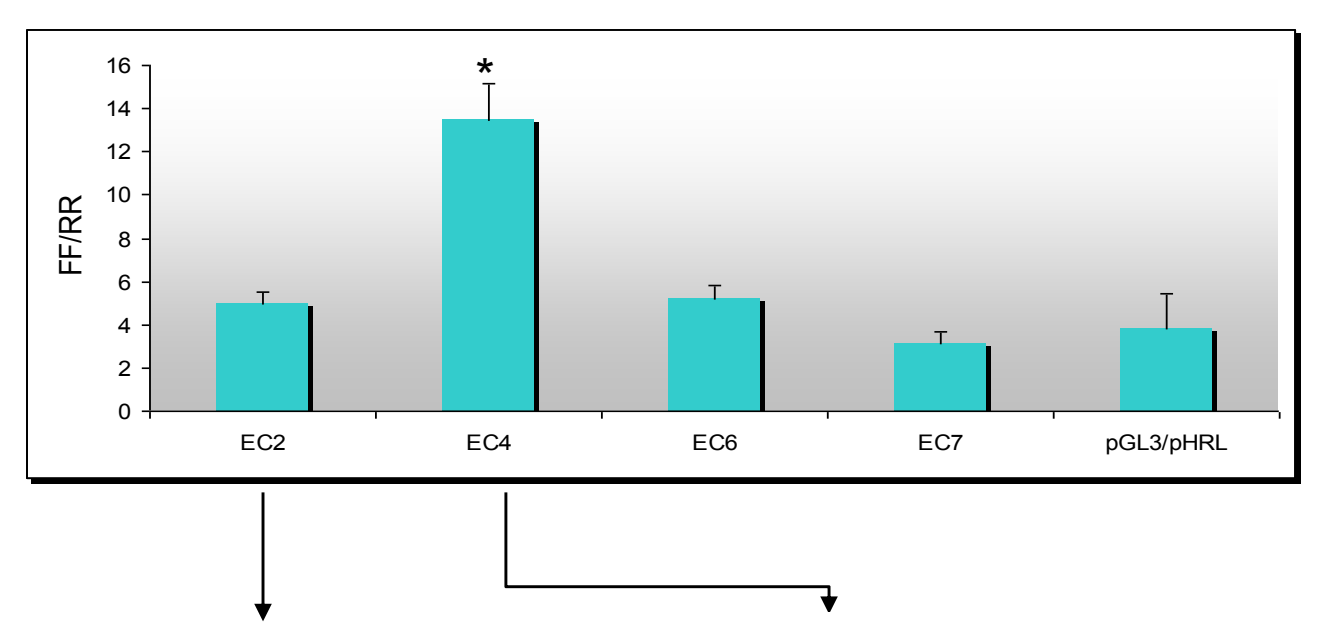

D

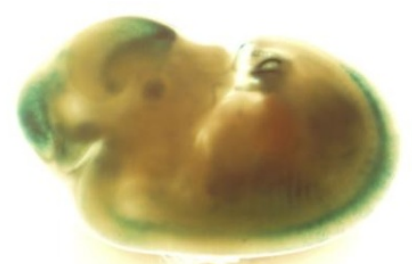

E13.5 (6/10)

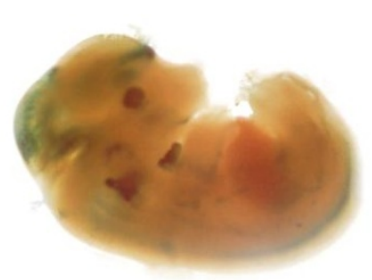

E13.5 (6/7)

Figure 6 Identification and functional validation of candidate regulatory elements. (A) Representation of the upstream region of Kcna 1, indicating the fragments tested in the chromosome conformation capture (3C) assays. (B) Graphic display of the conservation profiles for the upstream region of Kcnq1. The base genome is mouse. Evolutionarily conserved regions (ECRs) of a minimum of 100 bp conserved above $70 \%$ sequence identity are displayed as red (intergenic) peaks, with the $x$-axis representing positions in the base genome and the $y$-axis representing percentage identity between the base and the aligned genomes. Annotated genes are depicted in blue. Numbered peaks represent the

fragments tested for enhancer activity. (C) DNA sequences were inserted in pGL3-promoter vector upstream of the luciferase reporter. Luciferase activity was normalized to Renilla activity. All transfections were performed in triplicate. The asterisk denotes the interaction observed in neonatal heart by 3C. (D) Representative LacZ-stained embryos with in vivo enhancer activity. ECR4 and ECR2 exhibit tissue-specific activity, with numbers showing the reproducibility of LacZ reporter staining. 
differential histone modifications associated with repression $[20,21]$, and our data indicate that it is also devoid of DNA methylation on the paternal allele. We speculate that the intragenic enhancer may be marked in early embryogenesis by pioneer factors that maintain an open, poised chromatin status [22] and prevent assembly of repressive factors associated with the spreading of Kcnq1ot1 [4,5].

We propose that as development proceeds, both the upstream and downstream contacts evident in the $3 \mathrm{C}$ assays promote a conformation that excludes the Kcnq1 promoter from the influence of the Kcnq1ot1 ncRNA by establishing an autonomous regulatory loop (Figure 7A). An alternative is that tissue-specific enhancer-driven expression can override the silencing biochemically, by countering the chromatin condensation directed by Kcnq1ot1 (Figure 7B). In both cases, the DNA elements involved are altering chromatin conditions locally, thereby limiting a global long-range silencing mechanism; in other words, they are establishing a boundary. It will be crucial now to determine the DNA-binding factors that mediate this activity and the effect that deleting these regions has on the expression and imprinting patterns of Kcnq1 and neighboring genes. It will also be important to test if mutations that eliminate Kcnq1ot1 expression can affect the remodeling of the Kcnq1 locus during development. Additionally, it will be interesting to see whether genes silenced by different epigenetic mechanisms, either during development or in disease states, are governed by similar conformational determinants.

\section{Conclusions}

In summary, we have explored the organization of the Kcnq1 region in three-dimensional nuclear space during development and have identified DNA sequence elements that achieve the dual purpose of reactivating a gene silenced by a non-coding RNA and directing tissue-specific expression. Our studies underscore the importance of providing a three-dimensional context to the information on DNA methylation and chromatin modifications in order to fully grasp the dynamics of gene regulation in different cell lineages during development.
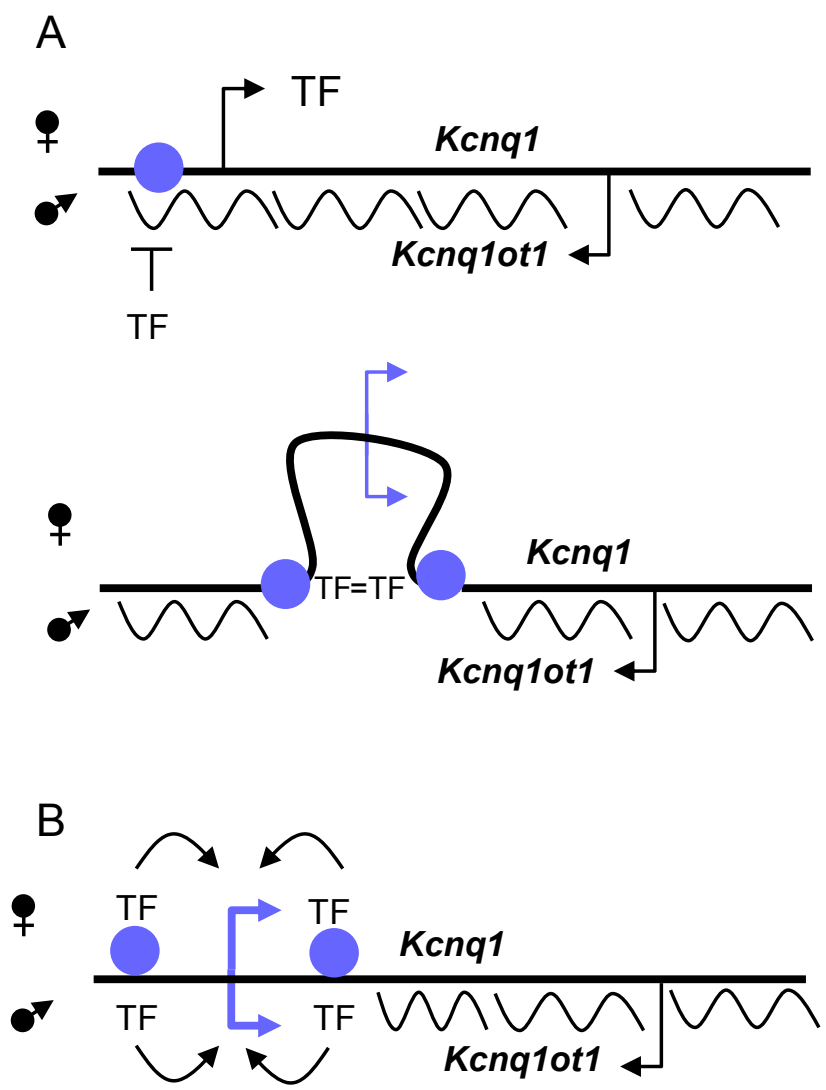

Figure 7 Model for escape from silencing by the Kcnq1 gene. Enhancer candidates, depicted as purple circles, become active upon binding tissue-specific transcription factors (TF); ncRNA, wavy lines. (A) Appearance of enhancer-specific transcription factors promotes a conformational change that sequesters the Kcnq1 promoter from the effects of Kcnq1ot1. (B) Factors binding in intron 1 of Kcnq1 act as a boundary for the Kcna10t1 ncRNA on the paternal allele, leaving it available for tissue-specific activation by enhancer-binding regulatory proteins. 


\section{Methods}

\section{Ethics}

This study was carried out on mice in strict accordance with the recommendations in the Guide for the Care and Use of Laboratory Animals of the National Institutes of Health. The protocol was approved by the Temple University Animal Care and Use Committee (Protocol 3294).

\section{RNA purification}

Heart tissues for total RNA extraction were collected at appropriate days of gestation using F1 hybrid mice from C57BL/6J $\times$ B6(CAST7) crosses from embryonic to neonatal stages. RNA was extracted using TRIzol Reagent (Invitrogen, Carlsbad, CA no. 15596-018) and following manufacturer's protocol for RNA extraction from tissues. All RNA samples were subjected to DNase treatment using Turbo DNA-free (Ambion, Austin, TX no. AM1907) using the rigorous DNase treatment protocol. Three to five biological samples were collected for each days of gestation.

\section{Reverse transcription}

Following the manufacturer's instructions, complementary DNA synthesis was performed on total RNA using SuperScript II Reverse Transcriptase (Invitrogen, Carlsbad CA, no. 18064-014). A reverse transcriptase negative control was used to ensure there was no DNA contamination.

\section{Real-time qRT-PCR}

Transcript levels of Kcnq1 and $\beta$-actin were analyzed on the ABI Prism 7000 system (ABI, Foster City, CA). Reactions were conducted using the SYBR Green PCR Master Mix (ABI, Foster City, CA no. 4309155). On a 96-well plate, a reduced reaction of $20 \mu \mathrm{l}$ was used instead of the suggested $50 \mu \mathrm{l}$ indicated in the manufacturer's protocol. This included $10 \mu \mathrm{l}$ of the SYBR Green reaction mix, a final concentration of $25 \mu \mathrm{M}$ of each primer, the cDNA template and water to bring the final volume up to $20 \mu \mathrm{l}$. The PCR was performed under the following conditions: an initial denaturing step for 10 min at $95^{\circ} \mathrm{C}$, an amplification step for 45 cycles of $95^{\circ} \mathrm{C}$ for $20 \mathrm{~s}, 55^{\circ} \mathrm{C}$ for $30 \mathrm{~s}$ and $72^{\circ} \mathrm{C}$ for $30 \mathrm{~s}$, the final elongation step was at $72^{\circ} \mathrm{C}$ for $2 \mathrm{~min}$. The Kcnq1 transcript was detected using the following left and right primers: 5'-CAAAGACCGTGGCAGTAAC-3' and 5'-CCTTCAT TGCTGGCTACAAC-3'. The Kcnq1 transcript was normalized to $\beta$-actin using the following left and right primers: 5'-TGTTACCAACTGGGACGACA-3' and 5'CCATCACAATGCCTGTGGTA-3'. Each qRT-PCR reaction was performed in triplicate with three biological replicates along with a no template negative control. The CT value of the Kcnq1 transcript was normalized to the CT value of the $\beta$-actin transcript. The standard deviation for each ratio was determined and the error bars represent $1 \mathrm{SD}$ away from the average ratio.

\section{Allele-specific RT-PCR and quantification}

The Kcnq1 transcript was amplified using Ruby Taq Master Mix (Affymetrix, Santa Clara, CA no. 71191) in a reduced $15 \mu \mathrm{l}$ reaction using the following primers: 5'CATCGGTGCCCGTCTGAACAGG-3' and 5'TTGCTGGGTAGGAAGAGCTCAG-3'. PCR reactions were performed with experimental and control templates in parallel. Following the PCR, a restriction digest was performed with the NlaIII (New England Biolabs, Ipswich, MA no. R0125) for $1 \mathrm{~h}$ at $37^{\circ} \mathrm{C}$. PCR and digestion products were run on $7 \%$ polyacrylamide gels and quantified using the Kodak Gel Logic 2000 imaging system (Kodak, Rochester, NY). The relative paternal to maternal band intensities were calculated.

\section{C assays}

$3 \mathrm{C}$ was performed as described previously with the following modifications: tissues were homogenized and crosslinked with $1 \%$ formaldehyde. Before restriction enzyme digestion, all samples were subjected to RNAse treatment with a combination of RNAse A and RNAse $\mathrm{H}$ at $37^{\circ} \mathrm{C}$ for 20 minutes. Restriction digests were carried out with $A f l \mathrm{III} / N c o$ I. Digestion efficiency at each restriction site was determined by comparing amplification with primers spanning the restriction site to amplification with primers immediately downstream on the digested template. No restriction bias was observed across the region assayed by $3 \mathrm{C}$ (data not shown). PCR efficiency of each combination of primers was assessed with a control template prepared from two bacterial artificial chromosomes (BACs) encompassing the Kcnq1ot1 region analyzed, as described previously. The linear range for each primer pair was determined by serial dilution. PCR reactions were performed with experimental and control templates in parallel and PCR products were run on $7 \%$ polyacrylamide gels and quantified using the Kodak Gel Logic 2000 imaging system (Kodak, Rochester, NY). Crosslinking frequencies were calculated from duplicate PCR analyses of three independent $3 \mathrm{C}$ preparations. Interaction frequencies for Kcnq1 and Kcnq1ot1 were normalized to a control interaction at the H19 locus to allow comparisons between different tissues and cell types. A full list of primers is available upon request from the authors.

\section{Luciferase reporter assays}

Enhancer candidates were amplified from mouse genomic DNA and subcloned into the pGL3-promoter vector (Promega, Madison, WI) upstream of the luciferase 
reporter. All clones were confirmed by sequencing. Constructs were screened by transfection of $100 \mathrm{ng}$ pGL3 promoter vector and $10 \mathrm{ng}$ pHRL basic vector/well (24well plate) into NIH 3T3 cells with $1 \mu$ l Lipofectamine 2000/well (Invitrogen, Carlsbad, CA). Cell lysate was harvested the following day and firefly and Renilla luciferase activities were measured in $10 \mu \mathrm{l}$ of each lysate using a Dual-Luciferase Reporter Assay System kit (Promega, Madison, WI) and firefly activity was normalized to Renilla. All transfections were performed in triplicate.

\section{Transgenic embryo assays}

Enhancer candidate constructs were amplified from mouse genomic DNA, subcloned into the Hsp68-LacZ vector and transgenic mouse embryos were generated and stained (Cyagen Biosciences, Sunvale, CA). Images were obtained with an Olympus MVX10 stereoscope (Olympus, Center Valley, PA), cropped and levels adjusted with Adobe Photoshop (Adobe, San Jose, CA).

\section{Methyl-DNA immunoprecipitation}

DNA from pooled mouse $7.5 \mathrm{dpc}$ embryos and from mouse sperm was sonicated to obtain fragments ranging from 200 to 1,000 bp. An aliquot of DNA was saved for an input positive control. Me-DIP was performed using the Methylamp Methylated DNA Capture Kit (Epigentek, Farmingdale, NY)) and following the manufacturer's protocol. The Kcnq1 CpG Island was amplified using the following: 5'-CTGAGGGCAGCACGGTCTAT-3' and 5'-CTCCTGAGTCTCTCTTGTCACAACT-3'. H19 and Kcnq1ot1 promoters were used to control the quality of the immunoprecipitated substrates.

\section{Acknowledgements}

We thank Natalie Sedlak and May Truong-Cao for technical support, and Keith Latham for comments on the manuscript. This work was supported by National Institutes of Health (grant K22CA140361-01).

\section{Authors' contributions \\ NE was responsible for the development of the experiments, the $3 \mathrm{C}$ assays and associated analysis, and wrote and edited the manuscript. LK performed the allele-specific RNA analysis, qRT-PCR analysis, MeDIP experiments and helped edit the manuscript. AR generated the $3 C$ substrates and helped perform the $3 C$ assays. GB performed and analyzed the dual luciferase assays. All authors read and approved the final manuscript}

\section{Competing interests}

The authors declare that they have no competing interests.

Received: 16 September 2011 Accepted: 15 November 2011 Published: 15 November 2011

\section{References}

1. Bartolomei MS: Genomic imprinting: employing and avoiding epigenetic processes. Genes Dev 2009, 23:2124-2133.

2. Wan LB, Bartolomei MS: Regulation of imprinting in clusters: noncoding RNAs versus insulators. Adv Genet 2008, 61:207-223.

3. Smilinich NJ, Day CD, Fitzpatrick GV, Caldwell GM, Lossie AC, Cooper PR, Smallwood AC, Joyce JA, Schofield PN, Reik W, Nicholls RD, Weksberg R,
Driscoll DJ, Maher ER, Shows TB, Higgins MJ: A maternally methylated CpG island in KvLQT1 is associated with an antisense paternal transcript and loss of imprinting in Beckwith-Wiedemann syndrome. Proc Natl Acad Sci USA 1999, 96:8064-8069.

4. Pandey RR, Mondal T, Mohammad F, Enroth S, Redrup L, Komorowski J, Nagano T, Mancini-Dinardo D, Kanduri C: Kcnq1ot1 antisense noncoding RNA mediates lineage-specific transcriptional silencing through chromatin-level regulation. Mol Cell 2008, 32:232-246.

5. Terranova R, Yokobayashi S, Stadler MB, Otte AP, van Lohuizen M, Orkin SH, Peters AH: Polycomb group proteins Ezh2 and Rnf2 direct genomic contraction and imprinted repression in early mouse embryos. Dev Cell 2008, 15:668-679.

6. Redrup L, Branco MR, Perdeaux ER, Krueger C, Lewis A, Santos F, Nagano T, Cobb BS, Fraser P, Reik W: The long noncoding RNA Kcnq1ot1 organises a lineage-specific nuclear domain for epigenetic gene silencing. Development 2009, 136:525-530.

7. Gould TD, Pfeifer K: Imprinting of mouse Kvlqt1 is developmentally regulated. Hum Mol Genet 1998, 7:483-487.

8. Dekker J, Rippe K, Dekker M, Kleckner N: Capturing chromosome conformation. Science 2002, 295:1306-1311.

9. Tolhuis B, Palstra RJ, Splinter E, Grosveld F, de Laat W: Looping and interaction between hypersensitive sites in the active beta-globin locus. Mol Cell 2002, 10:1453-1465.

10. Bokil NJ, Baisden JM, Radford DJ, Summers KM: Molecular genetics of long QT syndrome. Mol Genet Metab 2010, 101:1-8.

11. Kadauke $S$, Blobel GA: Chromatin loops in gene regulation. Biochim Biophys Acta 2009, 1789:17-25.

12. Tsai CL, Rowntree RK, Cohen DE, Lee JT: Higher order chromatin structure at the X-inactivation center via looping DNA. Dev Biol 2008, 319:416-425.

13. Engel N, Raval AK, Thorvaldsen JL, Bartolomei SM: Three-dimensional conformation at the H19/lgf2 locus supports a model of enhancer tracking. Hum Mol Genet 2008, 17:3021-3029.

14. Visel A, Minovitsky S, Dubchak I, Pennacchio LA: VISTA Enhancer Browsera database of tissue-specific human enhancers. Nucleic Acids Res 2007, 35: D88-92

15. Ovcharenko I, Nobrega MA, Loots GG, Stubbs L: ECR Browser: a tool for visualizing and accessing data from comparisons of multiple vertebrate genomes. Nucleic Acids Res 2004, 32:W280-286.

16. Mikkelsen TS, Ku M, Jaffe DB, Issac B, Lieberman E, Giannoukos G, Alvarez P, Brockman W, Kim TK, Koche RP, Lee W, Mendenhall E, O'Donovan A, Presser A, Russ C, Xie X, Meissner A, Wernig M, Jaenisch R, Nusbaum C, Lander ES, Bernstein BE: Genome-wide maps of chromatin state in pluripotent and lineage-committed cells. Nature 2007, 448:553-560.

17. Visel A, Blow MJ, Li Z, Zhang T, Akiyama JA, Holt A, Plajzer-Frick I, Shoukry M, Wright C, Chen F, Afzal V, Ren B, Rubin EM, Pennacchio LA: ChIP-seq accurately predicts tissue-specific activity of enhancers. Nature 2009, 457:854-858.

18. Filippova GN, Cheng MK, Moore JM, Truong JP, Hu YJ, Nguyen DK, Tsuchiya KD, Disteche CM: Boundaries between chromosomal domains of $\times$ inactivation and escape bind CTCF and lack CpG methylation during early development. Dev Cell 2005, 8:31-42.

19. Carrel L, Park C, Tyekucheva S, Dunn J, Chiaromonte F, Makova KD: Genomic environment predicts expression patterns on the human inactive $\times$ chromosome. PLoS Genet 2006, 2:e151.

20. Lewis A, Mitsuya K, Umlauf D, Smith P, Dean W, Walter J, Higgins M, Feil R, Reik W: Imprinting on distal chromosome 7 in the placenta involves repressive histone methylation independent of DNA methylation. Nat Genet 2004, 36:1291-1295.

21. Umlauf D, Goto Y, Cao R, Cerqueira F, Wagschal A, Zhang Y, Feil R: Imprinting along the Kcnq1 domain on mouse chromosome 7 involves repressive histone methylation and recruitment of Polycomb group complexes. Nat Genet 2004, 36:1296-1300.

22. Xu J, Watts JA, Pope SD, Gadue P, Kamps M, Plath K, Zaret KS, Smale ST: Transcriptional competence and the active marking of tissue-specific enhancers by defined transcription factors in embryonic and induced pluripotent stem cells. Genes Dev 2009, 23:2824-2838.

doi:10.1186/1756-8935-4-21

Cite this article as: Korostowski et al:: Enhancer-driven chromatin interactions during development promote escape from silencing by a long non-coding RNA. Epigenetics \& Chromatin 2011 4:21. 